\title{
Realization of Negative Permeability in Vertical Double-Split Ring Resonators with Normal Incidence
}

Hao-Yuan Tsai ${ }^{1,2,3}$, Che-Chin Chen ${ }^{2}$ *, Tse-An Chen ${ }^{1}$, Din Ping Tsai ${ }^{4}$, Takuo Tanaka ${ }^{3}$, and Ta-Jen

$\mathrm{Yen}^{1, *}$

Supporting Information

\section{Fabrication and curvature optimization via a Metal Stress Self-Folding Method}

(a)

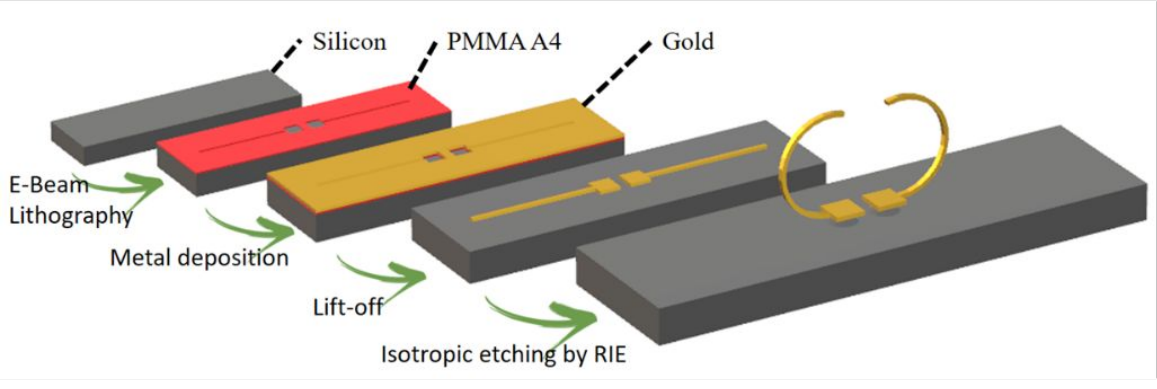

(b)

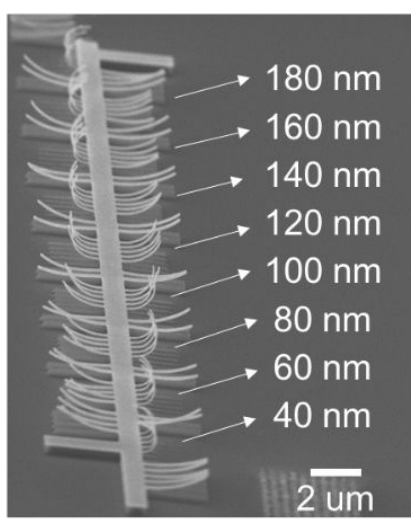

Figure S1. Illustration of the (a) fabrication process and the (b) the test-sample design for $\mathrm{Ni} / \mathrm{Au}(5 / 50 \mathrm{~nm})$ with thinner arm length from $40 \mathrm{~nm}$ to $180 \mathrm{~nm}$.

The detailed fabrication condition has been discussed in the METHOD section and the visualized process flow is shown in Figure $\mathrm{S} 1(\mathrm{a})$. The customized curvature can be designed the cantilever by different arms width and length with different etching recipe. We made test-sample for the 2-um-length-arms with different width from $20 \mathrm{~nm}$ to $180 \mathrm{~nm}$, as shown in Figure S1(b), for the isotropic-etching recipe optimization. And the results reveal 
that the structure with thinner arm width could have higher bended curvature.

\section{Comparison of Discrete and Connected 3D-DSRRs}
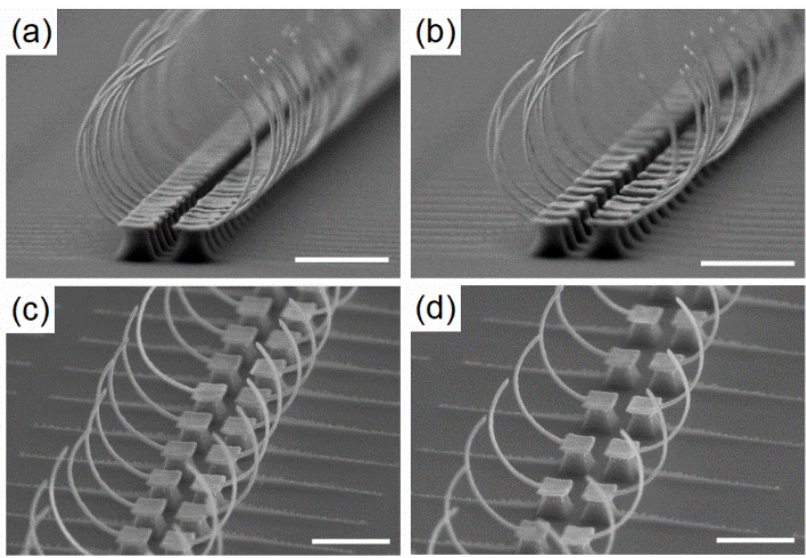

(d)

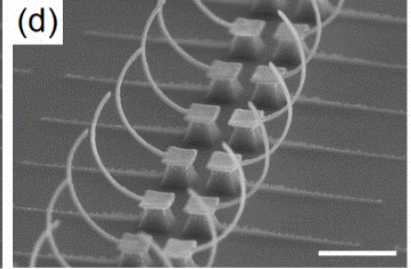

Figure S2. SEM images of discrete 3D-DSRRs: $(a, c)$ PX08 and (b, d) PX12, viewed at (a,

b) $85^{\circ}$ and (c, d) $60^{\circ}$. The white scale bars are $1 \mu \mathrm{m}$.
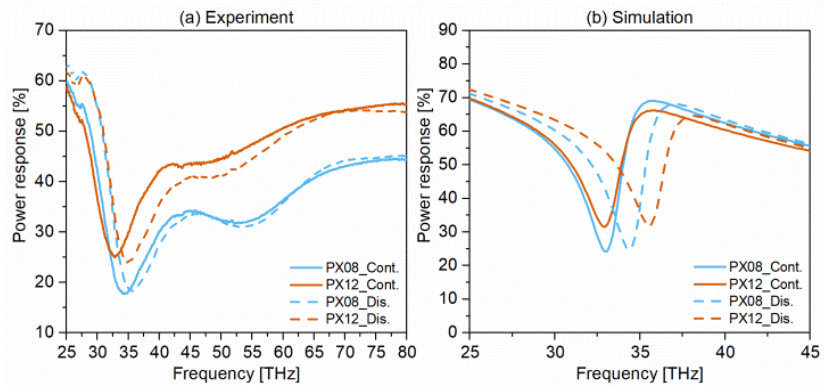

Figure S3. (a) Experimental and (b) simulated transmittance spectra of connected and discrete 3D-DSRRs, PX08 and PX12. For the experimental results, the out-of-resonance spectra are presented to confirm the absence of other modes that may affect the 3DDSRRs.

3. Bianisotropy Constitutive Parameter Retrieved from the Numerical S Parameter 
We followed the bianisotropic parameter retrieval method to perform postprocessing of our simulation results. We confirmed that the simulation results can match the assumption of a bianisotropic medium, in that the reflectance depends on the normal incidence for both the positive and negative directions and the transmittance remains constant due to a lack of inversion symmetry. We then output the complex S parameter as $r_{+}(\mathrm{S} 11), t_{+}(\mathrm{S} 21), t_{-}$ (S12), and $r_{-}(\mathrm{S} 22)$. The $(+)$ and $(-)$ signs in the equations refer to the propagation direction. According to the derivation, the normalized effective medium impedance can be expressed as follows:

$$
z_{ \pm}=\left(-b \mp \sqrt{b^{2}-4 a c}\right) /(2 a)
$$

with

$$
\begin{gathered}
\mathrm{a}=t_{+} t_{-}-\left(1-r_{+}\right)\left(1-r_{-}\right) \\
\mathrm{b}=\left(z_{1}-z_{2}\right)\left(t_{+} t_{-}+1-r_{+} r_{-}\right)+\left(z_{1}+z_{2}\right)\left(r_{+}-r_{-}\right) \\
\mathrm{c}=z_{1} z_{2}\left[-t_{+} t_{-}+\left(1+t_{+}\right)\left(1+r_{-}\right)\right]
\end{gathered}
$$

Here, $\mathrm{t}$ and $\mathrm{r}$ represent the complex transmission and reflection, and $z_{1}$ and $z_{2}$ give the medium impedance before and after the waves have passed through the metamaterial. The complex refractive index can be obtained by rewriting the impedance as follows:

$$
\cos \left(n k_{0} d_{S}\right)=\frac{t_{+}}{2}\left[\frac{1-z_{-} / z_{2}}{1+r_{+}-\left(1-r_{+}\right) z_{-} / z_{1}}+\frac{1-z_{+} / z_{2}}{1+r_{+}-\left(1-r_{+}\right) z_{+} / z_{1}}\right]
$$


where $k_{0}$ and $d_{s}$ are the vacuum wave vector and metamaterial slab thickness, respectively. (S.5) has an infinite number of solutions for complex values of $\mathrm{n}$ due to the branches of the inverse cosine. We chose the result that renders the constitutive parameters consistent with passive materials. Once $z_{ \pm}$and $\mathrm{n}$ are determined, we can deduce $\xi, \varepsilon$, and $\mu$ as follows:

$$
\begin{gathered}
\xi=\operatorname{in}\left(z_{-}+z_{+}\right) /\left(z_{-}-z_{+}\right) \\
\varepsilon=(n+i \xi) / z_{+} \\
\mu=z_{+}(n-i \xi)
\end{gathered}
$$

\section{Multipole Expansion with Numerical Current Density}

The multipole moment and radiation power can be obtained by integrating the simulated current density as follows:

Electric dipole moment and radiation power:

$$
\vec{P}=\frac{1}{i \omega} \int d^{3} \vec{j}, I_{p}=\frac{2 \omega^{4}}{3 c^{3}}|\vec{P}|^{2}
$$

Magnetic dipole moment and radiation power:

$$
\vec{M}=\frac{1}{2 c} \int d^{3} r(\vec{r} \times \vec{j}), \quad I_{m}=\frac{2 \omega^{4}}{3 c^{3}}|\vec{M}|^{2}
$$


Toroidal dipole moment and radiation power:

$$
\vec{T}=\frac{1}{2 c} \int d^{3} r\left[(\vec{r} \cdot \vec{j}) r-2 r^{2} \vec{j}\right], I_{T}=\frac{2 \omega^{6}}{3 c^{5}}|\vec{T}|^{2}
$$

Electric quadrupole moment and radiation power:

$$
\overrightarrow{Q_{\alpha \beta}}=\frac{1}{i 2 \omega} \int d^{3} r\left[r_{\alpha} j_{\beta}+r_{\beta} j_{\alpha}-\frac{2}{3} \delta_{\alpha \beta}(\vec{r} \cdot \vec{j})\right], I_{Q}=\frac{\omega^{6}}{5 c^{5}} \Sigma\left|\overrightarrow{Q_{\alpha \beta}}\right|^{2}
$$

Magnetic quadrupole moment and radiation power:

$$
\overrightarrow{M_{\alpha \beta}}=\frac{1}{3 c} \int d^{3} r\left[(\vec{r} \times \vec{j})_{\alpha} r_{\beta}+(\vec{r} \times \vec{j})_{\beta} r_{\alpha}\right], \quad I_{M}=\frac{\omega^{6}}{40 c^{5}} \Sigma\left|\overrightarrow{M_{\alpha \beta}}\right|^{2}
$$

where $\omega$ is the angular frequency, $c$ is the speed of light, $j$ is the current density at point $(x$, $y, z), r$ is the distance vector from the origin to $(x, y, z)$ in a Cartesian coordinate system, and $a, b$ represent the plane in which the quadrupole exists and is calculated.

Following the method described above, the multipole radiation powers of the series of $2 \mathrm{D}$ and 3D metamaterials were extracted, as shown in Figure S4; however, we focused on the electric and magnetic dipole radiation power in the main article to highlight the strong enhancement of the magnetic dipole moment radiation power obtained for the 3D-DSRRs. Figure S5 shows the magnetic field intensity and the current density under the frequency that the $2 \mathrm{D}$ and $3 \mathrm{D}$ structures have the maximum magnetic dipole radiation power. 

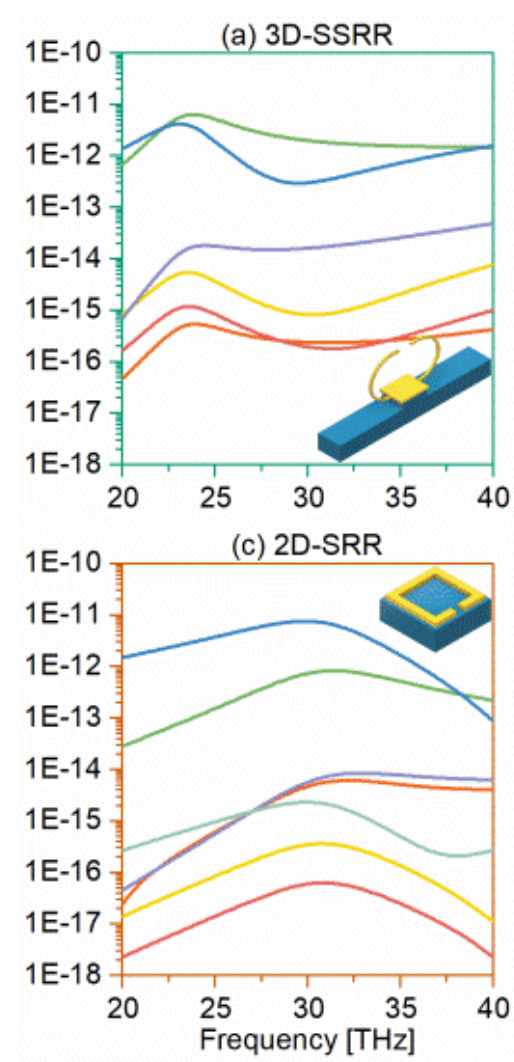

(b) 3D-DSRR

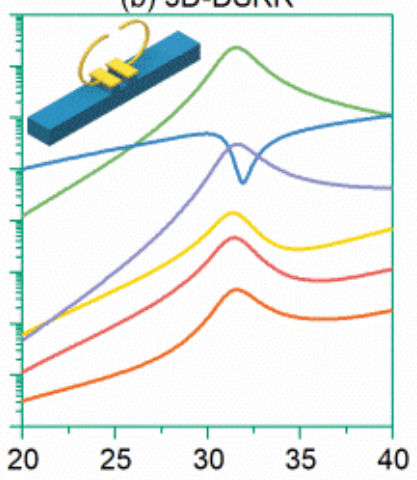

(d) Sym. 2D-DSRR

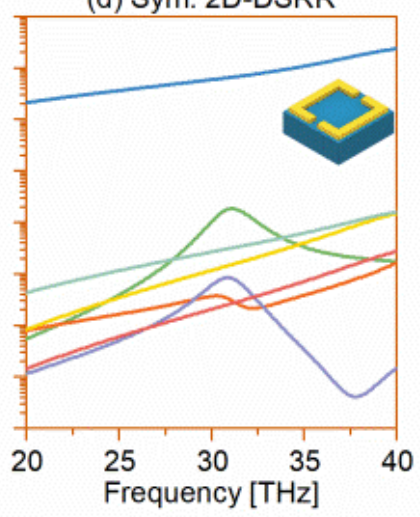

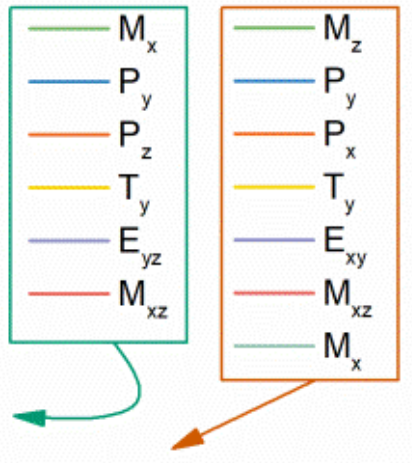

(e) Asym. 2D-DSRR

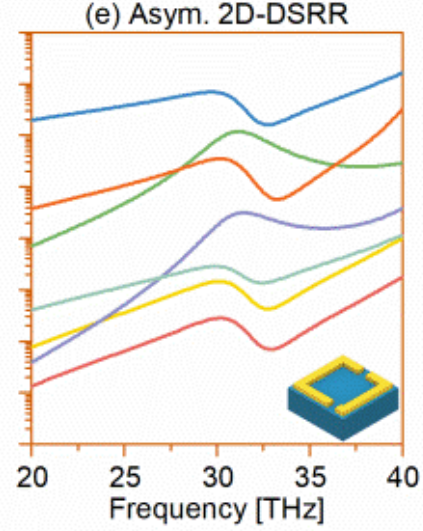

Figure S4. Multipole radiation power of the (a) 3D-SSRR, (b) 3D-DSRR, (c) 2D-SSRR, (d) symmetric 2D-DSRR, and (e) asymmetric 2D-DSRR. The insets display the geometries employed in the simulations. For a constant simulation boundary setup, normally incident, $x$-polarized illumination induced different multipoles for the 2D and 3D structures. 

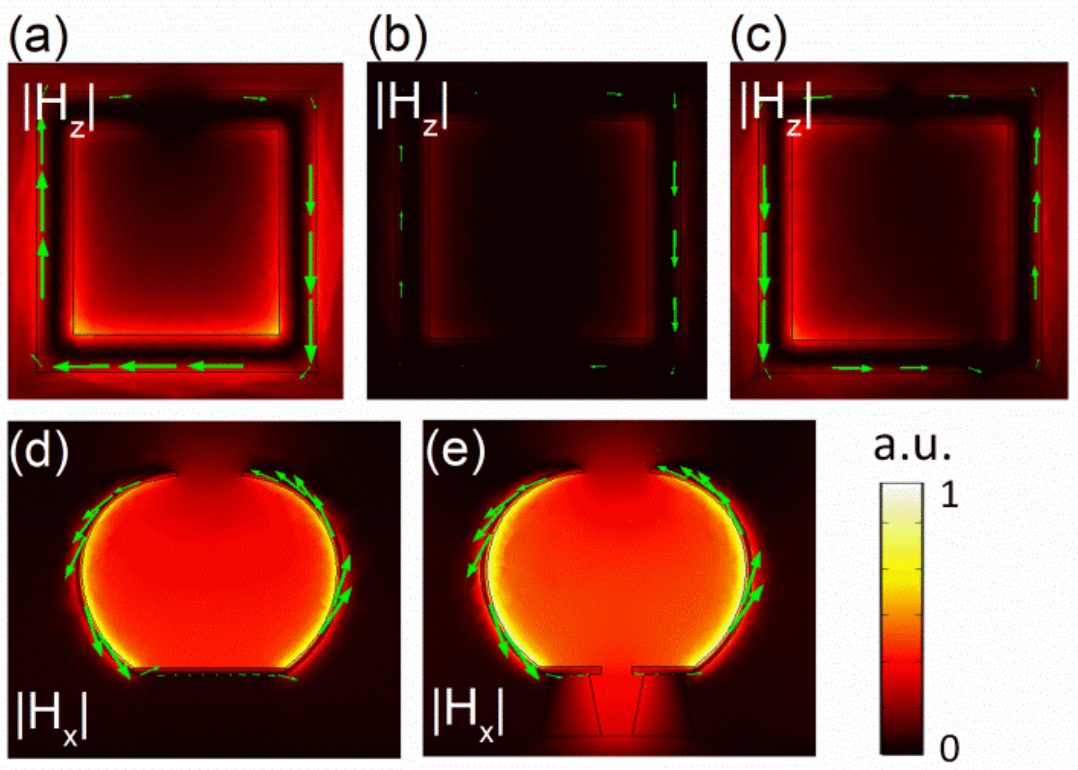

Figure S5. Magnetic field intensity and the current density vector of (a) 2D-SRR, (b) symmetric 2D-DSRR, (c) asymmetric 2D-DSRR, (d) 3D-SRR, (e) 3D-DSRR under resonance frequency along $(a-c) z$ axis and $(e, f)$ y axis under the resonance frequency. (ac) are the magnetic field intensity along $z$ axis of (a) 2D-SRR, (b) symmetric 2D-DSRR, and (c) asymmetric 2D-DSRR. $(d, e)$ are the magnetic field intensity along $x$ axis. 